\title{
Avelumab plus axitinib vs. sunitinib for advanced renal-cell carcinoma
}

\author{
Yue Zhang, Shenhong Wu \\ Division of Hematology and Oncology, Stony Brook University Hospital, Stony Brook, NY, USA \\ Correspondence to: Yue Zhang, MD, MPH. Assistant Professor of Medicine, Division of Hematology and Oncology, Stony Brook University Hospital, \\ HSC T15, RM 040, Stony Brook, NY 11794-8151, USA. Email: yue.zhang@stonybrookmedicine.edu. \\ Comment on: Motzer RJ, Penkov K, Haanen J, et al. Avelumab plus Axitinib vs. Sunitinib for Advanced Renal-Cell Carcinoma. N Engl J Med \\ 2019;380:1103-15.
}

Submitted Jun 07, 2019. Accepted for publication Jun 18, 2019.

doi: $10.21037 /$ tcr.2019.06.39

View this article at: http://dx.doi.org/10.21037/tcr.2019.06.39

Treatment for renal cell carcinoma (RCC) is undergoing landscape changes in recent years with introduction of immune check point inhibitors and combination regimens. Sunitinib and pazopanib are vascular endothelial growth factor receptor (VEGFR) tyrosine kinase inhibitors (TKIs). They have been the standard of care for the first line treatment of advanced RCC over a decade (1-3). Axitinib, sorafenib; the anti-VEGF monoclonal antibody bevacizumab; and the mammalian target of rapamycin (mTOR) inhibitors everolimus were often used in the second line or refractory setting with only 15-25\% response rate, significant toxicities and limited time for progression free survival (PFS) and overall survival (OS) (4-6). Avelumab plus axitinib combination is just approved for first-line treatment of patients with advanced RCC on May 14, 2019. This breakthrough offers RCC patients with another new treatment option and better outcome based on the result from a phase III, randomized, multicenter, openlabel JAVELIN Renal 101 trial (NCT02684006).

RCC is known to be immune sensitive with a record of interferon-alpha (IFN- $\alpha$ ) and high-dose interleukin (IL)-2 therapies as the standards of care till VEGFR TKIs in 2007. High-dose IL-2 is still used in selective advanced RCC patients due to around 7\% complete response and durable response (7). Immune checkpoint inhibitors such as anti-programmed cell death protein-1 (PD-1) and anti-programmed death-ligand 1 (PD-L1) monoclonal antibodies have made breakthroughs in multiple malignancies in recent years (8-11). Avelumab is a fully human mAb of the immunoglobulin (Ig) G1 isotype that specifically targets and blocks PD-L1. It has been approved for Merkel cell carcinoma and advanced urothelial carcinoma by FDA with multiple studies ongoing with a wide variety of malignancies as a single agent or in combination with treatment modalities (12-14). Immunotherapy with PD-1 inhibition has already proven to be effective in the second line and refractory setting with another PD-1 inhibitor nivolumab after VEGFR TKIs (15). The combination of nivolumab with an antibody to cytotoxic T-lymphocyte-associated protein 4 (CTLA-4) ipilimumab demonstrated acceptable safety and improved PFS and OS in intermediate or poor risk, previously untreated advanced RCC compared to sunitinib (16). Axitinib is an oral inhibitor of VEGF receptor and it was approved for advanced RCC after failure of sunitinib. A phase III clinical trial demonstrated axitinib significantly longer PFS compared with sorafenib for second-line therapy of advanced RCC. PFS was 6.7 months with axitinib compared to 4.7 months with sorafenib [hazard ratio (HR) 0.665 ; $95 \%$ CI: 0.544-0.812; one-sided $\mathrm{P}<0.0001]$ (4). Given the safety profile of lower risk of hepatic toxicities comparing to sunitinib and pazopanib, axitinib was chosen by the study group. Combining PD-L1 inhibitor avelumab with VEGFR TKI axitinib with the hope to increase response rate, prolong PFS and OS, and potential achieve durable response led to JAVELIN Renal 101 trial.

Eight hundred and eighty-six patients' treatment naïve advanced clear cell RCC regardless of tumor PD-L1 expression were randomized to receive either avelumab $10 \mathrm{mg} / \mathrm{kg}$ intravenous infusion every 2 weeks in combination with axitinib $5 \mathrm{mg}$ twice daily orally or sunitinib $50 \mathrm{mg}$ 
Table 1 FDA approval timeline for first line renal cell carcinoma

\begin{tabular}{ll}
\hline Favorable risk & Intermediate/poor risk \\
\hline High dose interleukin 2 [1992] & High dose interleukin 2 [1992] \\
Sunitinib [2006] & Sunitinib [2006] \\
Bevacizumab + interferon alfa & Temsirolimus [2007] \\
[2009] & \\
Pazopanib [2009] & Bevacizumab + interferon alfa \\
& [2009] \\
Axitinib + pembrolizumab [2019] & Pazopanib [2009] \\
Axitinib + avelumab [2019] & Carbozantinib [2016] \\
& Ipilimumab + nivolumab [2018] \\
& Axitinib + pembrolizumab \\
& [2019] \\
& Axitinib + avelumab [2019] \\
\hline
\end{tabular}

once daily orally for 4 weeks followed by 2 weeks off until radiographic progression or unacceptable toxicity. The primary outcomes were PFS, and OS in patients with PDL1-positive tumors. Five hundred and sixty patients with PD-L1-positive tumors (63.2\%) demonstrated a statistically significant improvement in PFS with 13.8 months in the avelumab plus axitinib combination cohort compared to 7.2 months in sunitinib (HR 0.61; 95\% CI: 0.47-0.79; $\mathrm{P}<0.0001)(17)$. Median PFS in the total population was also positive 13.8 vs. 8.4 months respectively (HR 0.69; 95\% CI: $0.56-0.84 ; \mathrm{P}<0.001)(17)$. With a median OS follow-up of 19 months, OS data were immature with $27 \%$ deaths in the intent-to-treat population. Among the patients with PD-L1-positive tumors, the response rate was $55.2 \%$ in avelumab plus axitinib cohort compared to $25.5 \%$ in sunitinib cohort; complete response rate were $4.4 \%$ vs. $2.1 \%$ respectively (17). PFS and OS favored combination in all subgroups assessed regardless of PD-L1 status and all Memorial Sloan Kettering Cancer Center (MSKCC) and International Metastatic Renal Cell Carcinoma Database Consortium (IMDC) prognostic risk groups. The frequency and severity of adverse events with the combination of avelumab plus axitinib were similar compared to sunitinib group, with $99.5 \%$ vs. $99.3 \%$ for any adverse event, $71.2 \%$ vs. $71.5 \%$ for grade 3 or higher in the respective groups (17). Avelumab plus axitinib as first-line treatment for advanced RCC is approved on May 14, 2019 based on the efficacy and safety profile from JAVELIN Renal 101 trial.

This is an exciting era for RCCs. The first line treatment for RCC dramatically improved with several options available.
Beyond avelumab plus axitinib approval, KEYNOTE-426 trial demonstrated level 1 evidence of PFS and OS benefit of another similar combination regimen with a PD-1 inhibitor pembrolizumab plus axitinib compared to sunitinib (survival rate $89.9 \%$ vs. $78.3 \%$; HR for death 0.53 ; $95 \%$ CI: $0.38-$ 0.74; $\mathrm{P}<0.0001$; PFS 15.1 vs. 11.1 months; HR 0.69; $95 \%$ CI: $0.57-0.84 ; \mathrm{P}<0.001)(18)$. The combination of a PD-1 inhibitor nivolumab with CTLA-4 antibody ipilimumab received approval based on OS and objective response rates improvement than with sunitinib among intermediateand poor-risk patients in first line setting too. The median OS was not reached with nivolumab plus ipilimumab $v s$. 26.0 months with sunitinib (HR 0.63; $\mathrm{P}<0.001$; OS $42 \%$ vs. $27 \%$. $\mathrm{P}<0.001)(16)$. Cabozantinib is an oral potent inhibitor of VEGFR2, MET, and AXL and also showed to significantly increased median PFS (8.2 vs. 5.6 months) and was associated with a $34 \%$ reduction in rate of progression or death (HR 0.66; 95\% CI: 0.46-0.95; one-sided $\mathrm{P}=0.012$ ). OR was $46 \%$ (95\% CI: $34-57 \%$ ) for cabozantinib vs. $18 \%$ (95\% CI: 10 $28 \%$ ) for sunitinib in a randomized phase II multicenter trial. It also gained FDA approval in patients with intermediate- or poor-risk RCC (19).

With the different options become available recently, the treatment paradigm for advanced RCCs has changed dramatically in the clinic (Table 1). How to select the treatment options in between the PD-1/PD-L1 inhibitor plus axitinib vs. PD-1 plus CTLA4? Is there still a role of sunitinib and pazopanib in the first line setting? Which patient population benefit more from cabozantinib? With the clear PFS and even OS benefit, single agent TKIs with either sunitinib or pazopanib are no longer the drug of preference for the first line use. However, the question remains whether or not immune check point inhibitor with TKI are synergistic or simply additive effect to make a sequential approach reasonable. There are no head to head comparison of the current approved combination regimens. Sunitinib has been used as the control arm for three immunotherapy combination studies. The PD-1 inhibitor and PD-L1 inhibitor did not show major difference in other studies in terms of efficacy and major toxicities so far (20). It is still an area under investigation. The combination of pembrolizumab and axitinib also already demonstrated OS benefit while avelumab and axitinib has not reach it. It is important to know the OS result when data matures with longer follow up. Multiple other combination regimens are also under evaluation such as atezolizumab plus bevacizumab vs. sunitinib; lenvatinib plus everolimus $v s$. lenvatinib plus pembrolizumab vs. sunitinib; nivolumab plus 
cabozantinib vs. sunitinib etc. The approach to combination regimens will become standard of care. It will be widely used in the first line setting with more data rising in the next several years.

IMDC model is widely used for risk stratification in both clinical trial and daily practice. The efficacy for the PD-1/PD-L1 inhibitor plus axitinib is across the board of favorable, intermediate and poor risk disease. However, nivolumab plus ipilimumab only demonstrated statistically significant improvement in patients with intermediate- or poor-risk RCC. The overall response rate in the favorable risk disease patients in contrast were much higher in sunitinib vs. the combination arm instead (52\% vs. $29 \%$; $\mathrm{P}=0.0002)$ as well as a significantly longer PFS (25.1 vs. 15.3 months; $\mathrm{P}<0.001)(16)$. VEGFR inhibition seems to have an important role in the favorable risk disease group. However, one caveat is the much higher complete response rate of $9 \%$ with nivolumab plus ipilimumab compared to $5.8 \%$ and $3.4 \%$ complete response rate in the pembrolizumab and avelumab plus axitinib trials respectively (16-18). cabozantinib was also only approved for intermediateor poor-risk RCC based on phase II clinical trial. Risk classification needs to be done with each individual newly diagnosed advanced RCC patient. It should be discussed with patient and their family. Individual patient's preference should be taken into consideration for regimen selection.

PD-L1 expression status has been assessed in all the immunotherapy trials. The combination of avelumab and axitinib trial even selected patients with PD-L1 positive for PFS and OS analysis as primary end points. However, the three trials used different assays for their evaluations. In the exploratory analysis for nivolumab plus ipilimumab trial showed longer PFS was observed with patients with $1 \%$ or greater PD-L1 expression but not in the negative PD-L1 group, while OS and response rate (RR) was not affected by PD-L1 expression status. The benefits of pembrolizumab plus axitinib with respect to OS and PFS were observed in all subgroups examined regardless of PD-L1 expression status. PD-L1 expression as a biomarker for these immunotherapies is unsatisfactory with limitations in both daily clinical practice and hurdles in clinical trial interpretation. With the immunotherapy-based combination regimens approval one after another, the economic burden requires more attention. More effectively select patients who will benefit from these treatments is critical important. Better biomarker is an unmet need to further classify advanced RCC and more effectively predict treatment response to immunotherapy and/or VEGFR inhibitors.
In the era of cancer immunotherapy, avelumab and axitinib combination is a brand-new addition to the first line treatment options for RCC. It is not only practice changing but also advance the landscape of combination immunotherapy and target therapy.

\section{Acknowledgments}

Funding: None.

\section{Footnote}

Provenance and Peer Review: This article is commissioned and reviewed by the Section Editor Hong-chao He (Department of Urology, Shanghai Ruijin Hospital, Shanghai Jiaotong University School of Medicine, Shanghai, China).

Conflicts of Interest: Both authors have completed the ICMJE uniform disclosure form (available at http://dx.doi. org/10.21037/tcr.2019.06.39). The authors have no conflicts of interest to declare.

Ethical Statement: The authors are accountable for all aspects of the work in ensuring that questions related to the accuracy or integrity of any part of the work are appropriately investigated and resolved.

Open Access Statement: This is an Open Access article distributed in accordance with the Creative Commons Attribution-NonCommercial-NoDerivs 4.0 International License (CC BY-NC-ND 4.0), which permits the noncommercial replication and distribution of the article with the strict proviso that no changes or edits are made and the original work is properly cited (including links to both the formal publication through the relevant DOI and the license). See: https://creativecommons.org/licenses/by-nc-nd/4.0/.

\section{References}

1. Motzer RJ, Hutson TE, Tomczak P, et al. Sunitinib versus interferon alfa in metastatic renal-cell carcinoma. $\mathrm{N}$ Engl J Med 2007;356:115-24.

2. Botta GP, Granowicz E, Costantini C. Advances on immunotherapy in genitourinary and renal cell carcinoma. Transl Cancer Res 2017;6:17-29.

3. Motzer RJ, Hutson TE, Cella D, et al. Pazopanib versus sunitinib in metastatic renal-cell carcinoma. $\mathrm{N}$ Engl J Med 2013;369:722-31. 
4. Rini BI, Escudier B, Tomczak P, et al. Comparative effectiveness of axitinib versus sorafenib in advanced renal cell carcinoma (AXIS): a randomised phase 3 trial. Lancet 2011;378:1931-9.

5. Yang JC, Haworth L, Sherry RM, et al. A randomized trial of bevacizumab, an anti-vascular endothelial growth factor antibody, for metastatic renal cancer. N Engl J Med 2003;349:427-34.

6. Lucarelli G, Ferro M, Ditonno P, et al. The urea cycle enzymes act as metabolic suppressors in clear cell renal cell carcinoma. Transl Cancer Res 2018;7:S766-S9.

7. Belldegrun AS, Klatte T, Shuch B, et al. Cancer-specific survival outcomes among patients treated during the cytokine era of kidney cancer (1989-2005): a benchmark for emerging targeted cancer therapies. Cancer 2008;113:2457-63.

8. Weber J, Mandala M, Del Vecchio M, et al. Adjuvant Nivolumab versus Ipilimumab in Resected Stage III or IV Melanoma. N Engl J Med 2017;377:1824-35.

9. Eggermont AMM, Blank CU, Mandala M, et al. Adjuvant Pembrolizumab versus Placebo in Resected Stage III Melanoma. N Engl J Med 2018;378:1789-801.

10. Gandhi L, Rodriguez-Abreu D, Gadgeel S, et al. Pembrolizumab plus Chemotherapy in Metastatic NonSmall-Cell Lung Cancer. N Engl J Med 2018;378:2078-92.

11. Le DT, Uram JN, Wang H, et al. PD-1 Blockade in Tumors with Mismatch-Repair Deficiency. N Engl J Med 2015;372:2509-20.

12. Fehrenbacher L, Spira A, Ballinger M, et al. Atezolizumab versus docetaxel for patients with previously treated non-small-cell lung cancer (POPLAR): a multicentre, open-label, phase 2 randomised controlled trial. Lancet

Cite this article as: Zhang $\mathrm{Y}, \mathrm{Wu} \mathrm{S}$. Avelumab plus axitinib vs. sunitinib for advanced renal-cell carcinoma. Transl Cancer Res 2019;8(Suppl 6):S585-S588. doi: 10.21037/tcr.2019.06.39
2016;387:1837-46.

13. Kaufman HL, Russell J, Hamid O, et al. Avelumab in patients with chemotherapy-refractory metastatic Merkel cell carcinoma: a multicentre, single-group, open-label, phase 2 trial. Lancet Oncol 2016;17:1374-85.

14. Rosenberg JE, Hoffman-Censits J, Powles T, et al. Atezolizumab in patients with locally advanced and metastatic urothelial carcinoma who have progressed following treatment with platinum-based chemotherapy: a single-arm, multicentre, phase 2 trial. Lancet 2016;387:1909-20.

15. Motzer RJ, Escudier B, McDermott DF, et al. Nivolumab versus Everolimus in Advanced Renal-Cell Carcinoma. N Engl J Med 2015;373:1803-13.

16. Motzer RJ, Tannir NM, McDermott DF, et al. Nivolumab plus Ipilimumab versus Sunitinib in Advanced Renal-Cell Carcinoma. N Engl J Med 2018;378:1277-90.

17. Motzer RJ, Penkov K, Haanen J, et al. Avelumab plus Axitinib versus Sunitinib for Advanced Renal-Cell Carcinoma. N Engl J Med 2019;380:1103-15.

18. Rini BI, Plimack ER, Stus V, et al. Pembrolizumab plus Axitinib versus Sunitinib for Advanced Renal-Cell Carcinoma. N Engl J Med 2019;380:1116-27.

19. Choueiri TK, Halabi S, Sanford BL, et al. Cabozantinib Versus Sunitinib As Initial Targeted Therapy for Patients With Metastatic Renal Cell Carcinoma of Poor or Intermediate Risk: The Alliance A031203 CABOSUN Trial. J Clin Oncol 2017;35:591-7.

20. Alsaab HO, Sau S, Alzhrani R, et al. PD-1 and PDL1 Checkpoint Signaling Inhibition for Cancer Immunotherapy: Mechanism, Combinations, and Clinical Outcome. Front Pharmacol 2017;8:561. 